\title{
Reprodutibilidade do questionário Transporte Ativo e rotinas em adolescentes portugueses Reproducibilidad del cuestionario de Transporte Activo y rutinas en adolescentes portugueses Reproducibility of the Active Transport questionnaire and routines in Portuguese adolescents *Ana Paula Matos, **Eduarda Maria Coelho, *Maria Beatriz Pereira, ***Sergio Souza *Universidade do Minho (Portugal), **University of Trás-os-Montes and Alto Douro (Portugal), ***Universidade de São Luís do Maranhão
} (Brasil)

Resumo. O transporte ativo casa-escola é incentivado por vários organismos nacionais e internacionais visando a promoção da saúde. No entanto, em Portugal não se identificou nenhum estudo de validação de um questionário sobre este tipo de transporte para jovens. O objetivo deste trabalho é avaliar a estabilidade temporal do questionário «Transporte Ativo e Rotinas», após um período de 4 semanas em uma amostra de adolescentes portugueses, constituída por 43 crianças (15 Masc. e 23 Fem.) com idades compreendidas entre 9-12 anos (10,5 \pm 0,6). O questionário «Transporte Ativo e Rotinas,» é um instrumento desenvolvido no Instituto de Educação (Centro de Investigação em Estudos da Criança) da Universidade do Minho. Na determinação dos níveis de reprodutibilidade, utilizou-se o procedimento de teste e reteste, com intervalo de 4 semanas entre as duas aplicações. O questionário mostra uma confiabilidade aceitável, verificada por meio de consistência temporal, designadamente em questões relativas à caracterização geral; utilização da bicicleta, caminhada, colegas e família; e segurança da bicicleta. No que diz respeito a questões relacionadas com a saúde e autonomia e meio ambiente já não se aplica. Este estudo preliminar, mostra que o questionário «Transporte Ativo e Rotinas» apresenta uma fiabilidade aceitável, verificada por meio de consistência temporal. O questionário pode ser replicado, mas deve ser reduzido (mantendo apenas os três primeiros grupos de perguntas), alterado no vocabulário, adaptando-o à faixa etária em estudo e visualmente mais atraente.

Palavras-chave: Reprodutibilidade; questionário; transporte ativo; adolescentes.

Resumen. El propósito de este artículo es evaluar la estabilidad temporal del cuestionario «Transporte Activo y Rutinas», después de un período de 4 semanas en una muestra de adolescentes portugueses. La muestra de 43 niños (15 Masc. y 23 Fem.), con edades entre 9-12 años (10,5 \pm 0,6). El cuestionario «Transporte Activo y Rutinas» es un instrumento desarrollado en el Instituto de Educación (Centro de Investigación para Estudios del Niño) de la Universidad do Minho. En la determinación de los niveles de reproducibilidad, se utilizó el procedimiento de prueba y prueba, 4 semanas de intervalo entre las dos aplicaciones. El cuestionario muestra una fiabilidad aceptable, verificado por consistencia temporal, particularmente en cuestiones relativas a la caracterización general; uso de la bicicleta, a pie, compañeros y familiares; y la seguridad en bicicleta. Con respecto a los temas relacionados con la salud y la autonomía y el medio ambiente y el ahorro de la misma ya no se aplica. Este estudio preliminar muestra que el cuestionario «Transporte Activo y Rutinas», presenta una fiabilidad aceptable, verificado por consistencia temporal. El cuestionario se puede replicar, pero debe ser de tamaño reducido (manteniendo sólo los tres primeros grupos de preguntas) cambiando el vocabulario adaptado al grupo de edad en estudio y el uso de una presentación más atractiva visualmente.

Palabras clave: Reproducibilidad; cuestionario; transporte activo; adolescentes.

Abstract. Active transportation between home and school is encouraged by several international and national organizations with the aim to promote health. However, no study on the validation of questionnaires on this type of transportation in youth has been carried out in Portugal to the date. The purpose of this study was to assess the external validity of the questionnaire «Active Transportation and Routines» during a 4-week period in a sample of 43 Portuguese adolescents (15 boys and 23 girls) aged 9 to 12 years (10,5 \pm 0,6). The questionnaire «Active Transportation and Routines» was developed by the Institute of Education (Research Center for the Studies on Children) of the University of Minho. In order to evaluate external validity, a test-retest procedure was used with a lapse of 4-weeks between the two applications. The instrument shows an acceptable reliability, verified through temporal consistency on the dimensions of general characterization, use of bicycle, walking, peers and family, and safety on bicycle. Regarding the items related with health and autonomy and environment, this questionnaire is not used for investigating these topics any longer. This preliminary study shows that the questionnaire «Active Transportation and Routines» is reliable and consistent over time. The instrument can be replicated; however, it should be shortened (keeping only the first three groups of items), its vocabulary might be changed in order to adapt it according to the age range it is used for, and it could be visually more attractive.

Keywords: Repeatability; questionnaire; active transportation; adolescents.

\section{Introdução}

Grande parte da investigação existente sobre atitudes em relação ao transporte sustentável refere-se a adultos. Pouco se sabe sobre as atitudes dos jovens em relação à sustentabilidade, particularmente em relação à escolha do transporte, e como estas afetam o seu comportamento. Assistimos a uma cultura de uso do carro, alimentada por um medo e aversão dos ambientes naturais e as respostas dos pais sobre a segurança dos filhos à custa do desenvolvimento da sua independência, apesar das crianças expressarem atitudes positivas em relação ao transporte sustentável dando-nos assim uma perspetiva de conflito (Lorenc, Brunton, Oliver, Oliver, \& Oakley, 2008). A criança necessita de tempo e de espaço para brincar, de forma livre e espontânea, necessita sentir segurança nas atividades de brincadeira que realiza, para que, em simultâneo, se desenvolvam mecanismos mentais de segurança emocional e íntima. A independência de mobilidade é entendida como a capacidade de autonomia, ou seja, a possibilidade de tomar decisões por si própria face ao envolvimento físico, das «possibilidades de ação» que

Fecha recepción: 15-12-16. Fecha de aceptación: 14-06-17 Ana Paula Matos

paularmatoso@yahoo.com.br a criança está capaz de realizar. Esta possibilidade, de ser e estar capaz de «se movimentar e deslocar» no espaço, permite-lhe «pensar e agir» em função dessa experiência. Permite-lhe um Ser, um Eu próprio (Neto \& Malho, 2007) . Esta perda de mobilidade interfere diretamente no estado de saúde da criança (Hu et al., 2003; Sofi, Capalbo, Cesari, Abbate, \& Gensini, 2008). A criança apresenta uma necessidade fisiológica de se movimentar, se esta fica comprometida, compromete o desenvolvimento motor e funcional da criança, colocando em risco a qualidade futura de seunível de saúde(Machado-Rodrigues et al., 2014). O deslocamento fisicamente ativo para escola pode constituir uma importante fonte de atividade física diária (Tudor-Locke,Ainsworth, \& Popkin, 2001). Evidências consistentes sugerem que a prática de atividade física nos deslocamentos estáintimamente associada a diversos desfechos relacionados à saúde, como o aumento dos níveis gerais de atividade física (Kowalski, Crocker, \& Faulkner, 1997) de aptidão cardiorrespiratória (Cooper et al., 2006), manutenção da massa corporal (Heelan et al., 2005) (Hamer \& Chida, 2008) e redução do risco de acidente vascular cerebral isquémico (Hu G. et al., 1994).

A nível da literatura internacional Davison, Davison, Reed, Halden \& Dillon (2002) no seu trabalho «Children's Attitudes to Sustainable Transport» apresentam três questionários, para alunos do ensino básico, secundário e um outro para encarregados de educação sobre trans- 
porte casa/escola. Este projeto, tem como objetivo, compreender as atitudes das crianças e jovens relativamente ao transporte sustentável. A pesquisa, destacou a importância de compreender as diferenças de idade, gênero e localização. Hillman, Adams, and Whitelegg (1990) o seu estudo «Children's independent mobility: a comparative study in England and Germany (1971-2010)» utilizaram questionários para alunos e encarregados de educação. Estes, utilizados em 1971, 1990 e 2010 foram muito semelhantes e realizados em sala de aula. Villa González (2011) na província de Granada-Espanha, no seu estudo «Factores personales y ambientales asociados con el desplazamiento activo al colegio de los escolares españoles» inquiriu 745 crianças através de um questionário sobre transporte ativo contemplando as seguintes questões: «Como te deslocas para a escola? A pé, carro, bicicleta, motocicleta ou autocarro « Essa resposta foi categorizada em: ativos (a pé ou bicicleta) e passivos (carro, motocicleta ou autocarro). Ainda em Espanha, surge o estudo «Importancia del desplazamiento activo al colegio en la salud de los escolares espanoles» de Ortega et al. (2005) retirado do estudo AVENA* («alimentación y valoración del estado nutricional en adolescentes»). A iniciativa deste projeto de pesquisa transversal, surge na sequência dos maus hábitos alimentares, sedentarismo e síndromes como a anorexia e bulimia nervosa, que estão a afetar cada vez mais e mais cedo a população juvenil Espanhola. Foram inquiridos 2.859 jovens entre os 13 a 18 anos. A avaliação do comportamento em relação à atividade física e desportiva, condicionada por atitudes, motivações e valores, éfeita a partir de um questionário desenvolvido especificadamente para o estudo AVENA, que sofreu um processo de validação e confiabilidade ao estudar diferentes idades.

Da recolha bibliográfica realizada, encontramos também vários questionários que contemplam questões relativas ao transporte ativo, integrado em questionários sobre atividade física, como o questionário Global School-Based Health Survey (Global GSHS), proposto pela Organização Mundial da Saúde (OMS). A pesquisa da saúde do aluno na escola, é um projeto de colaboração para ajudar os países a medir e avaliar os fatores de risco comportamentais e fatores de proteção em 10 áreas: (1) informações pessoais; (2) consumo de álcool e drogas; (3) hábitos alimentares; (4) higiene; (5) sentimentos e relacionamentos; (6) atividades físicas; (7) comportamentos na escola; 8) comportamento sexual; (9) tabagismo e (10) violência entre os jovens, com idades compreendidas entre os 13 e os 17 anos. O Questionário Internacional de Atividade Física (IPAQ), no domínio dois explora as atividades físicas como o meio de transporte. Este domínio, é composto por três questões tendo como objetivo verificar como se deslocam os adolescentes numa semana normal, incluindo todos os meios de transporte (autocarro, carro, moto, bicicleta...) e a pé (não incluir as caminhadas de lazer e como exercício físico).

No que diz respeito a Portugal, o uso da bicicleta enquanto meio de transporte surgiu da preocupação de uma equipa de trabalho da Universidade do Minho (Instituto de Educação), sendo o fio condutor de toda a pesquisa. Associada a esta problemática, estava a falta de autonomia que as crianças apresentam na sua deslocação diária para a escola, bem como, a mobilidade como um aspeto crítico do desenvolvimento das crianças e a sua qualidade de vida. Foi criada, uma comissão de avaliação na Universidade do Minho - Instituto da Criança, que através de reuniões quinzenais decidiram fazer um levantamento das opiniões das crianças sobre o uso da bicicleta (novembro-dezembro 2012). A maior parte das questões para elaboração da entrevista e posterior questionário foram retiradas do programa ConBici (ConBici, 2007).

O resultado do primeiro inquérito/entrevista aplicado por Pereira, et al. (2013a) a alguns alunos de duas turmas, numa escola do concelho de Viana do Castelo cujo tema foi «Abicicleta como meio de transporte para a escola-recetividade e perceções dos alunos» permitiu ver questões relacionadas ao desejo dos estudantes para se deslocarem de bicicleta para a escola, com restrições diante da preocupação dos pais; dos perigos do trânsito, do receio em ir só e da transpiração que essa atividade física poderia causar. Desse levantamento preliminar, ficou evidente que o Projeto deverá prever a participação direta e ativa da família. A seguir Pereira, et al. (2013b) aplicaram a primeira versão do questionário a duas turmas de uma escola de Braga ( $\left(5^{\circ}\right.$ e $6^{\circ}$ anos). Depois de aplicado já se retificaram questões que estavam menos percetíveis e outras acrescentadas. Foi também elaborado um questionário para os pais, uma vez que são os principais decisores relativamente ao transporte dos educandos, que a seguir à primeira aplicação sofreu reformulações, uniformizando e aproximando os dois questionários do Educando e do Encarregado de Educação. Em 2013/14, o questionário foi aplicado por seis professores estagiários em cinco escolas diferentes: Sampaio (2014), Sousa (2014), Ferreira (2014), Leite (2014), Cardoso (2014) e Lopes (2014) nas suas teses demestrado. Os questionários (aluno e encarregado de educação) foram aplicados ao ensino secundário, em escolas do concelho de Guimarães. Foram feitas alterações ao questionário no sentido de o melhorar, deixou de ser em Word passou a ser em Excel tornando-o mais atrativo visualmente e consequentemente mais fácil de responder. Em 2014/15 é aplicado por três professores, direcionados para o $5^{\circ}$ e $6^{\circ}$ ano e $8^{\circ}$ ano, são eles: Costa (2015) , L. Costa (2015) e Cunha (2015).

Atualmente, o questionário está aprovado pela DGE (Direção de Serviços de Projetos Educativos) com o n. ${ }^{\circ} 0101600009$, com a designação «OTransporte Ativo de Bicicleta nos Hábitos de Deslocação para a Escola», registado em 03-02-2014, retratando hábitos de deslocação para a escola, nomeadamente o recurso ao transporte ativo de bicicleta como alternativa ao automóvel. O questionário está aprovado também pelo Comitê de Ética para Pesquisas Envolvendo Seres Humanos, da Universidade do Minho.

\section{Material e Métodos}

\section{Participantes}

Participaram neste estudo 43 adolescentes (15 Masc. e 23 Fem.), com idades compreendidas entre 9-12 anos $(10,5 \pm 0,6)$, de duas turmas de uma escola pública de Braga. Uma turma de $5^{\circ}$ ano com 17 alunos e outra de $6^{\circ}$ ano com 26 alunos (como se observa na Tabela 1). É uma amostra por conveniência.

\section{Instrumento}

O questionário «Transporte Ativo e Rotinas» é um instrumento específico que foi desenvolvido no Instituto (Universidade do Minho) com a finalidade de auxiliar professores, encarregados de educação e população em geral que trabalham com a prevenção, para que estes possam conhecer e medir melhor o estilo de vida dos seus alunos/filhos, nomeadamente na mobilidade independente das crianças, ou capacitar para a «liberdade dos alunos/filhos viajarem em torno de seu próprio bairro ou cidade, sem supervisão deum adulto». O conceito demobilidade independente estáintimamente relacionado com a autonomia das crianças na exploração do ambiente físico disponível. Tentamos criar a capacidade de a criança desenvolver «imagens mentais» dos lugares. Se a criança está capaz de se situar a partir do «sítio em que reside», está a ser capaz de «criar» identificações de lugares porque as suas vivências permitem «desenvolver sentimentos de pertença» (Neto \& Malho, 2007) . O conteúdo do questionário divide-se em cinco áreas de modo a simplificar a sua estrutura e facilitar o seu preenchimento, são elas: I) Caraterização geral quanto à distância, tempo e modo de deslocação casa/ escola., tendo sido as quatro questões associadas a este item retiradas do Instituto Nacional de Estatística - Censos 2011 (Estatistica, 2011); II) Uso da bicicleta, pares e família; III) Bicicleta em segurança, IV) Saúde, autonomia e V) Ambiente e poupança. Este questionário contém questões abertas onde solicitamos opiniões ou informações, especialmente úteis para reações exploratórias ou subjetivas e questões fechadas de escolha múltipla ou de respostas curta, resposta direta.

\section{Desenho da investigação e procedimentos}

O questionário construído e reformulado ao longo de três anos foi submetido à apreciação de dois especialistas, doutores, profissionais da área da atividade física e do desporto; que têm experiência em metodologia de pesquisa; e concordaram em participar na avaliação do instrumento. 
Para a verificação da reprodutibilidade do instrumento utilizou-se o procedimento teste-reteste, tendo sido realizadas duas recolhas de dados ao mesmo grupo de alunos, com intervalo de quatro semanas. A aplicação do instrumento foi efetuada por um único avaliador, nas duas aplicações. Para verificar a clareza, o questionário foi aplicado a quarenta e três alunos, aos quais foi solicitado que se manifestassem se a questão não estivesse clara ou se tivessem dificuldade de perceber. Após os procedimentos iniciais, onde foi solicitado autorização ao Agrupamento de Escolas e enviada uma carta de informação aos Encarregados de Educação e o termo de consentimento livree esclarecido para ser assinado, o questionário foi aplicado a primeira vez dia seis de novembro numa aula deEducação Física de uma escola pública de Braga. Os questionários levaram aproximadamente 15 minutos a serem respondidos. As dúvidas que surgiram foram: não sabem a profissão do Encarregado deEducação; não distinguem desporto federado de lazer; não sabem a distância casaescola, assim como, o tempo gasto no percurso; alegam também que umas vezes utilizam um meio de transporte e em dias de chuva usam outro. Na questão 23, não sabem o significado do termo «finalidade»; na questão 36 não percebem o que é uma «norma» e na questão 47 não sabem o queé «autonomia». Nas questões abertas, muitas vezes preferem colocar «não» para não terem de explanar. A segunda aplicação fez-se passada quatro semanas, a quatro de dezembro. O questionário foi aplicado exatamente às mesmas turmas, no mesmo espaço. As questões onde tiveram dúvidas foram: continuam a não saber a profissão e nível de escolaridade do Encarregado de Educação, não distinguem desporto federado edelazer e continuam ater dificuldades nas palavras «finalidade» e «autonomia».

\section{Análise Estatística}

Para a análise estatística dos dados foi utilizado o programa SPSS 22 (Statistical Package for the Social Sciences). Na elaboração de tabelas foi utilizado o programa informático Microsoft Office Excel 2010.

A análise da reprodutibilidade teste-reteste foi efetuada utilizando o Índice Kappa (K). Segundo a proposta de (Landis \& Koch, 1977), o coeficiente Kappa foi classificado em: mau (0); pobre $(0,01$ a 0,20$)$; regular (0,21 a 0,40); moderado (0,41 a 0,60); substancial $(0,61$ a 0,80$)$; e quase-perfeito ou excelente $(>0,80)$. O limite máximo de ê é 1 , representando o acordo perfeito entre avaliações. Por outro lado, quanto mais próximo de 0 estiver o valor de ê, mais este sugere que o grau de acordo se deve ao acaso (Fonseca, Silva, \& Silva, 2013).

\section{Resultados}

\section{Dados Sócio Biográficos da Amostra}

\begin{tabular}{lccc}
$\begin{array}{l}\text { Tabela } 1 \\
\text { Dados Sócio Biográficos da Amostra }\end{array}$ & & & \\
\hline \multirow{2}{*}{ Sexo } & Masculino & 15 & $\%$ \\
\hline \multirow{4}{*}{ Idade } & Feminino & 28 & 34,9 \\
& 9 anos & 2 & 65,1 \\
\hline \multirow{2}{*}{ Ano/Escolaridade } & 10 anos & 18 & 41,7 \\
& 11 anos & 21 & 48,8 \\
& 12 anos & 2 & 4,7 \\
\hline \multirow{2}{*}{ Encarregado Educação (EE) } & $5^{\circ}$ ano & 17 & 39,5 \\
& $6^{\circ}$ ano & 26 & 60,5 \\
\hline \multirow{2}{*}{ EE Trabalha? } & Pai & 9 & 20,9 \\
& Mãe & 34 & 79,1 \\
\hline \multirow{2}{*}{ Setor Profissão EE } & Sim & 36 & 83,7 \\
& Não & 7 & 16,3 \\
\hline \multirow{2}{*}{ Nível de Estudos EE } & Setor Secundário & 5 & 14,3 \\
& Setor Terciário & 30 & 85,7 \\
\hline & $1^{\circ}$ ciclo & 1 & 2,4 \\
& $2^{\circ}$ ciclo & 5 & 11,9 \\
& $3^{\circ}$ ciclo & 3 & 7,1 \\
& Secundário & 6 & 14,3 \\
EE - Encarregado de Educação & Licenciatura & 10 & 23,8 \\
& Mestrado/Doutorado & 1 & 2,4 \\
& Não Sei & 16 & 38,1 \\
\hline & & &
\end{tabular}

Da análise do quadro, podemos realçar o seguinte: a maior parte dos Encarregados deEducação são as mães (79,1\%), destas 83,7\% trabalham, principalmente no sector terciário (85,7\%) e 23,8\% são licenciadas.

\section{Reprodutibilidade do Questionário}

Tabela 2

Dados do Questionário

\begin{tabular}{|c|c|c|c|c|}
\hline & Kappa & Cat.* & $p$ & $\% \mathrm{C}$ \\
\hline Distância casa-escola & 0,422 & MO & 0 & 56 \\
\hline Transporte mais utilizado para escola & 0,691 & SU & 0 & 82,9 \\
\hline Utilizas um $2^{\circ}$ meio transporte & 0,773 & SU & 0 & 88 \\
\hline Quanto tempo demoras a chegar à escola? & 0,863 & $\mathrm{QP}$ & 0 & 97,5 \\
\hline Sabes andar de bicicleta? & 0,64 & SU & 0 & 95 \\
\hline $\begin{array}{l}\text { Gostarias de ir a pé ou de bicicleta para a escola se os amigos o } \\
\text { fizessem? }\end{array}$ & 0,24 & $\mathrm{RE}$ & 0,053 & 88,3 \\
\hline $\begin{array}{l}\text { Gostarias de andar de bicicleta se alguém de que gostas muito o } \\
\text { fizesse? }\end{array}$ & 0,393 & $\mathrm{RE}$ & 0,002 & 88,3 \\
\hline Tens bicicleta? & 0,79 & SU & 0 & 97,6 \\
\hline Se sim, com que finalidade utilizas? & 0,552 & MO & 0 & 86 \\
\hline Como gostarias de vir para a escola? & 0,288 & $\mathrm{RE}$ & 0,005 & 72 \\
\hline Quantas vezes gostarias de vir a pé ou de bicicleta para a escola? & 0,25 & $\mathrm{RE}$ & 0,022 & 67 \\
\hline $\begin{array}{l}\text { Pensa ser possível fazer o percurso casa-escola a pé ou de } \\
\text { bicicleta? }\end{array}$ & 0,4 & $\mathrm{RE}$ & 0,006 & 69,7 \\
\hline Costumas andar de bicicletas com familiares? & 0,808 & $\mathrm{QP}$ & 0 & 95 \\
\hline Se sim, com quem? & 0,616 & SU & 0 & 69,7 \\
\hline Se sim, com que periodicidade? & 0,515 & MO & 0 & 39,5 \\
\hline Andas de bicicleta? & 0,777 & SU & 0 & 95,3 \\
\hline Se sim, sente segurança e respeito quando circulas de bicicleta? & 0,626 & SU & 0 & 90,6 \\
\hline Pensas existir algum trajeto alternativo mais seguro? & 0,083 & PO & 0,516 & 53,4 \\
\hline Conhece as regras de circulação e conduta a pé ou de bicicleta? & 0,498 & MO & 0 & 81,3 \\
\hline Quando andas de bicicleta, respeitas as regras? & 0,311 & $\mathrm{RE}$ & 0,002 & 83,7 \\
\hline A tua escola possui estacionamento para bicicletas? & 0,143 & PO & 0,077 & 88,3 \\
\hline O estacionamento para bicicletas é seguro? & 0,294 & $\mathrm{RE}$ & 0,007 & 67,4 \\
\hline A escola dinamiza atividades desportivas de bicicleta ou a pé? & 0,233 & $\mathrm{RE}$ & 0,072 & 67,4 \\
\hline Se sim, costumas participar? & 0,249 & $\mathrm{RE}$ & 0,026 & 69,7 \\
\hline Sabes que a bicicleta permite transportar pequenos objetos? & 0,407 & MO & 0,002 & 81,3 \\
\hline Sabes fazer pequenos arranjos na bicicleta? & 0,826 & $\mathrm{QP}$ & 0 & 90,6 \\
\hline $\begin{array}{l}\text { A utilização da bicicleta e o andar a pé no percurso casa-escola é } \\
\text { importante para a tua saúde? }\end{array}$ & 0,656 & SU & 0 & 97,6 \\
\hline $\begin{array}{l}\text { A utilização da bicicleta e o andar a pé no lazer é importante para } \\
\text { a tua saúde? }\end{array}$ & $-0,87$ & Má & 0,565 & 83,7 \\
\hline $\begin{array}{l}\text { A utilização da bicicleta e o andar a pé no percurso casa-escola é } \\
\text { importante para a tua autonomia? }\end{array}$ & $-0,036$ & Má & 0,762 & 90,6 \\
\hline $\begin{array}{l}\text { A utilização da bicicleta poderia reduzir o tempo gasto no } \\
\text { percurso casa-escola? }\end{array}$ & $-0,08$ & Má & 0,555 & 46,5 \\
\hline $\begin{array}{l}\text { A utilização da bicicleta ou andar a pé ajudariam a poupar } \\
\text { dinheiro? }\end{array}$ & 0,476 & MO & 0,002 & 95,3 \\
\hline $\begin{array}{l}\text { A utilização da bicicleta e o andar a pé seriam importantes para } \\
\text { reduzir o trânsito? }\end{array}$ & $-0,032$ & Má & 0,823 & 93 \\
\hline
\end{tabular}

\%C: Coeficiente de Concordância

Na dimensão referente à «caracterização geral», os índices de concordância Kappa oscilaram entre 0,422 (moderados) para «Distância casa-escola» a 0,863 (quase perfeita) para «Quanto tempo demoras a chegar à escola?». Quando se faz a média dos valores do Índice Kappa para este conjunto de questões o valor obtido é 0,687 .

Nas questões referentes ao «uso de bicicleta, deslocar a pé, pares e família», os índices Kappa variaram entre regulares a quase perfeitos. Kappa de 0,240 para «Gostarias de ir a pé ou de bicicleta para a escola se os amigos o fizessem?» e de 0,808 para «Costumas andar de bicicletas com familiares?». Todas as outras questões apresentaram valores de Kappa médios. Quando se faz a média dos valores do Índice Kappa para este conjunto de questões o valor obtido é 0,499.

Na dimensão referente à «utilização da bicicleta em segurança», os valores de Kappa oscilaram entre 0,083 (pobre) para «Pensas existir algum trajeto alternativo mais seguro?» a 0,826 (quase perfeita) para «Sabes fazer pequenos arranjos na bicicleta?». Existem ainda duas questões que apresentam um valor de Kappa não significativo: «A tua escola possui estacionamento para bicicletas?» e «A escola dinamiza atividades desportivas de bicicleta ou a pé?». Quando se faz a média dos valores do Índice Kappa para este conjunto de questões o valor obtido é 0,404 .

Nas questões relativas à «saúde e autonomia» existem dois itens que apresentam uma má concordância «A utilização da bicicleta e o andar a pé no lazer é importante para a tua saúde?» e «A utilização da bicicleta e o andar a pé no percurso casa-escola é importante para a tua autonomia?» e um item uma concordância substancial («A utilização da bicicleta e o andar a pé no percurso casa-escola é importante para a tua saúde»).

Nas questões relativas ao «ambiente e poupança» existem dois itens que apresentam uma má concordância «A utilização da bicicleta poderia reduzir o tempo gasto no percurso casa-escola?» e «A utilização da bicicleta e o andar a pé seriam importantes para reduzir o trânsito?» 
eum item com uma concordância moderada ( «A utilização da bicicleta ou andar a pé ajudariam a poupar dinheiro?»).

\section{Discussão}

O transporte ativo casa-escola pode ser considerado uma forma de atividade física para os jovens (Cooper, Andersen, Wedderkopp, Page, \& Froberg, 2005), sendo incentivado como forma de promoção da saúde, por vários organismos nacionais e internacionais. Não foi identificado em Portugal qualquer estudo de validação de um questionário sobre este tipo de transporte em jovens, sendo necessário analisar alguns indicadores relativos às propriedades psicométricas do Questionário Transporte Ativo e Rotinas para este instrumento poder ser utilizado em Portugal.

Neste estudo verificou-se que o Questionário Transporte Ativo e Rotinas apresenta níveis moderados de reprodutibilidade para esta amostra. No que diz respeito aos fatores «Caracterização Geral» e «Uso da Bicicleta, Deslocar a Pé, Pares e Família» a reprodutibilidadeé elevada, variando o valor de K de Cohen entre 0,240 - 0,863. Relativamente à utilização da «Bicicleta em Segurança» $(0,083$ - 0,826), «Saúde e Autonomia» $(-0,870-0,656)$ e «Ambiente e Poupança» $(-0,08$ 0,476 ), existem itens em que a reprodutibilidade é elevada e outros em que é pobre. Talvez isto se deva ao facto do questionário ser demasiado longo, pois verifica-se uma menor fiabilidade nas questões finais. Por outro lado, a utilização de uma terminologia não compreendida pela maior parte dos adolescentes, poderá também justificar estes resultados. O questionário contem palavras que os adolescentes não compreenderam, como «finalidade», «normas», «autonomia» e «periodicidade». O elevado tempo que distanciou as duas aplicações do instrumento também pode explicar que algumas questões tenham uma fiabilidade reduzida, pois a opinião dos jovens se alterar.

Quando analisamos o questionário na globalidade, verificamos que 9,4\% das questões apresentam uma fiabilidade perfeita (3/32), 25\% substancial (8/32), 18,8\% moderada(6/32), 28,1\% regular (9/32), 6,3\% pobre (2/32)e 12,5\% má (4/32). Um estudo de validação para a India de um questionário para avaliação da distância e forma das crianças se deslocarem para a escola, obteve valores de Kappa semelhantes (Zaccari \& Council, 2003). Numa investigação realizada por Evenson, et al., (2008) , acerca da fiabilidade teste-reteste do questionário de School Travel Survey (avalia a viajem para e da escola: modo, companhia, destino depois da escola) verificaram um acordo substancial entre as duas aplicações para todas as questões. Os valores de Kappa foram superiores aos obtidos no nosso estudo o que pode ser justificado pelas duas aplicações dos questionários serem em dias seguidos, que faz com que os indivíduos se possam lembrar das respostas dadas. Um estudo de verificação da fiabilidade de um instrumento acerca das barreiras à deslocação ativa para a escola, em Espanha, obteve valores de Kappa superiores aos obtidos no nosso estudo, entre 0,230 («haver muito tráfego») e 0,693 («estar longe») (Garcia et al., 2017). Estes valores de Kappa superiores aos obtidos no nosso estudo podem ser justificados pela distância temporal entre as duas aplicações, que são inferiores à do nosso estudo, que faz com que os indivíduos se possam lembrar de algumas das respostas dadas.

Além deste estudo contribuir para a validação e desenvolvimento de um instrumento para avaliar o transporte casa-escola em Língua Portuguesa, deverá ser realçado também o facto de avaliar a perceção da distância e tempo que a criança demora nesta deslocação. No entanto, existem algumas limitações que deveremos ter em conta. Primeiro, não é possível generalizar os resultados obtidos devido a ter sido utilizada uma amostra de conveniência e a faixa etária ser reduzida, devendo ser realizados mais estudos deste género em indivíduos mais velhos. Também, é de sugerir a verificação da validade de critério, através da verificação da concordância entre as respostas dos pais e filhos. Estas informações podem orientar o planeamento e a avaliação de programas educativos, semelhantes ao Safe Routes to School, Kids Walk-to-school, PediBus ou CicloBus, que favorecem a utilização de meios de transporte fisicamente ativo para ir à escola e voltar para casa.

\section{Agradecimento}

Este trabalho foi financiado por Fundos Nacionais através da FCT (Fundação para a Ciência e a Tecnologia) e cofinanciado pelo Fundo Europeu de Desenvolvimento Regional (FEDER) através do COMPETE 2020 - Programa Operacional Competitividade e Internacionalização (POCI) no âmbito do CIEC (Centro de Investigação em Estudos da Criança da Universidade do Minho) com a referência POCI-01-0145-FEDER-007562

\section{Conclusões}

Este estudo preliminar mostra que o Questionário Transporte Ativo e Rotinas apresenta uma fiabilidade aceitável, verificada por meio de consistência temporal. O questionário pode ser utilizado, mas deve ser reduzido (mantendo apenas os três primeiros grupos de perguntas), alterado o vocabulário, adaptando-o à faixa etária, e tornado visualmente mais atraente. São necessários mais estudos de validação noutras idades e que realizem outros tipos de validação, como a validade de critério.

O mesmo já se encontra disponível em formato digital.

\section{Referências}

Ashley R. Cooper, Niels Wedderkopp, Han Wang, Lars Bo Andersen, Karsten Froberg,, \& Page, Angie S. (2006). Active Travel to School and Cardiovascular Fitness in Danish Children and Adolescents. Medicine \& Science in Sports \& Exercise , 0195-9131/06/3810 1724/0. doi: 10.1249/01.mss.0000229570.02037.1d

Bradshaw, Ruth, Lane, Robert, Tanner, Graham, \& Wofinden, Diana (1998). Levels of activity relating to safer routes to school type projects and green transport plans. Transport Studies Group of the University of Westminster.

Cardoso (2014). Transporte ativo para a escola e os fatores socioeconómicos: um estudo com alunos do ensino secundário.Tese de Mestrado, Instituto da Criança, Universidade do Minho.

ConBici (2007). Con Bici Al Cole-Proyecto pedagógico para primária. www.conbicialcole.com. Consultado a: 14 Jan 2016

Cooper, Ashley R,Andersen, Lars Bo, Wedderkopp, Niels, Page, Angie S, \& Froberg, Karsten (2005). Physical activity levels of children who walk, cycle, or are driven to school. American journal of preventive medicine, 29(3), 179-184.

Costa (2015). Transporte ativo: comparação dos padrões de deslocação casa-escola dos alunos do meio rural com o dos alunos do meio urbano. Tese de Mestrado, Instituto da Criança, Universidade do Minho.

Costa (2015). Transporte ativo entre casa e escola: estudo de uma escola urbana de $2^{\circ}$ ciclo. Tese de Mestrado, Instituto da Criança, Universidade do Minho.

Cunha (2015). Transporte ativo em alunos do $5^{\circ} e 6^{\circ}$ ano de escolaridade, por género, em meio predominantemente rural. Tese de Mestrado, Instituto da Criança, Universidade do Minho.

Davison, Paul, Davison, Philippa, Reed, Nick, Halden, Derek, \& Dillon, Justin (2002). Children's attitudes to sustainable transport: Scottish Executive, Social Research.

Estatistica, Instituto Nacional de. (2011). Censos 2011. http:// c e n s o s. i n e. p t / x p o r t a l/ xmain?xpid=CENSOS\&xpgid=censos2011_apresentacao, consultado a: 25 maio 2016

Ferreira (2014). Transporte ativo para a escola: a sua influência, na saúde e relação com a aptidão aeróbica de alunos do $11^{\circ}$ e 12 anos: relatório de estágio. Tese de Mestrado, Instituto da Criança, Universidade do Minho.

Fonseca, Ricardo, Silva, Pedro, \& Silva, Rita (2013). Acordo interjuízes: o caso do coeficiente kappa. Laboratório de Psicologia, 5(1), 81-90.

Garcia, Manuel Perez, Gutierrez, Sara Hermoso, Ruiz, Jonathan Ruiz, \& Garzón, Palma Chillón (2017). Fiabilidad de un cuestionario de 
barreras del desplazamiento activo al colegio (Reliability of a questionnaire about barriers to active travel to school). Retos(32), 72-75.

Hamer, Mark, \& Chida, Yoichi (2008). Active commuting and cardiovascular risk: a meta-analytic review. Preventive medicine, 46(1), 9-13.

Heelan, Kate A, Donnelly, Joseph E, Jacobsen, Dennis J, Mayo, Matthew S, Washburn, Rik, \& Greene, Lori (2005). Active commuting to and from school and BMI in elementary school children-preliminary data. Child: care, health and development, 31(3), 341-349.

Hillman, Mayer, Adams, John, \& Whitelegg, John. (1990). One false move. London: Policy Studies Institute.

Hu, Gang, Qiao, Qing, Silventoinen, K, Eriksson, Johan G Jousilahti, Pekka, Lindström, Jaana, . . . Tuomilehto, Jaakko. (2003). Occupational, commuting, and leisure-time physical activity in relation to risk for type 2 diabetes in middle-aged Finnish men and women. Diabetologia, 46(3), 322-329.

Kowalski, KentC, Crocker, PR, \& Faulkner, RobertA. (1997). Validation sf the physical activity questionnaire for older children. Pediatric exercise science, 9(4), 174-186.

Landis, J Richard, \& Koch, Gary G. (1977). The measurement of observer agreement for categorical data. biometrics, 159-174.

Leite (2014). Análise do meio circunjacente e relação como transporte ativo para a escola: um estudo em adolescentes do $11^{\circ} \mathrm{e} 12^{\circ}$ ano de escolaridade numa escola secundária. Tese de Mestrado, Instituto da Criança, Universidade do Minho.

Lopes (2014). Transporte ativo: implementação e avaliação de um programa de intervenção sobre os hábitos de deslocação em bicicleta para a escola. Tese de Mestrado, Instituto da Criança, Universidade do Minho.

Lorenc, Theo, Brunton, Ginny, Oliver, Sandy, Oliver, Kathryn, \& Oakley, Ann. (2008). Attitudes to walking and cycling among children, young people and parents: a systematic review. Journal of epidemiology and community health, 62(10), 852-857.

Machado-Rodrigues, Aristides M, Santana, Ana, Gama, Augusta, Mourão, Isabel, Nogueira, Helena, Rosado, Victor, . . . Padez,
Cristina (2014). Active commuting and its associations with blood pressure and adiposity markers in children. Preventive medicine, 69, 132-134.

Neto, C, \& Malho, MJ. (2007). Espaço urbano e a independência de mobilidadena infância, 2004.

Ortega, Francisco B, Ruiz, Jonatan R, Castillo, Manuel J, Moreno, Luis A, González-Gross, Marcela, Wärnberg, Julia, . . . Group, Avena (2005). Low level of physical fitness in Spanish adolescents. Relevance for future cardiovascular health (AVENAstudy). Revista Española de Cardiología (English Edition), 58(8), 898-909.

Pereira, Beatriz Oliveira, Silva, Inês Peixoto, Monteiro, Rita João F, Farenzena, Rosana, \& Rosário, Rafaela. (2013 a,b). Transporte ativo nas rotinas de vida das crianças: estudo em escola urbana.

Sampaio (2014). Transporte ativo: perceção e comportamentos de segurança no percurso de casa-escola de jovens do $11^{\circ} \mathrm{e} 12^{\circ}$ anos: relatório de estágio. Tese de Mestrado, Instituto da Criança, Universidade do Minho.

Sofi, Francesco, Capalbo, Andrea, Cesari, Francesca, Abbate, Rosanna, \& Gensini, Gian Franco (2008). Physical activity during leisure time and primary prevention of coronary heart disease: an updated meta-analysis of cohort studies. European Journal of Cardiovascular Prevention \& Rehabilitation, 15(3), 247-257.

Sousa (2014). Transporte ativo para a escola e a sua relação com o IMC: um estudo em adolescentes de uma escola secundária de Guimarães: relatório de estágio. Tese de Mestrado, Instituto da Criança, Universidade do Minho.

Szklo, Moyses, Nieto, F Javier, \& Miller, David. (2001). Epidemiology: beyond the basics. American Journal of Epidemiology, 153(8), 821-822.

Tudor-Locke, Catrine, Ainsworth, Barbara E, \& Popkin, Barry M. (2001). Active commuting to school. Sports Medicine, 31(5), 309313.

Villa González, Emilio (2011). Factores personales y ambientales asociados con el desplazamiento activo al colegio de los escolares españoles.

Zaccari, Vera, \& Council, Leichhardt (2003). Walking To School For Better Heath And Safety.

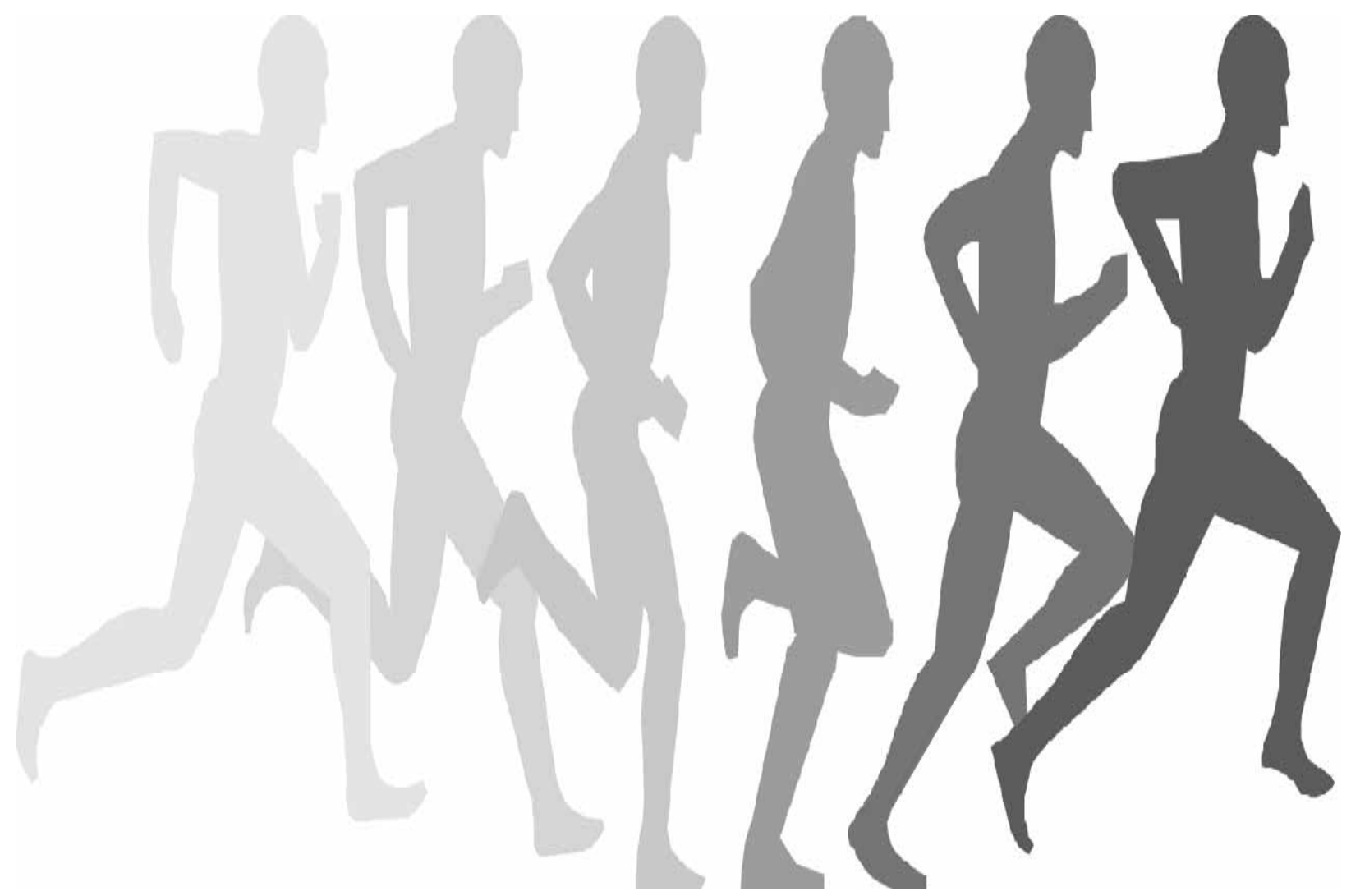

